\title{
Slowly-growing gap-opening planets trigger weaker vortices
}

\author{
Michael Hammer, ${ }^{1 \star}$ Kaitlin M. Kratter ${ }^{1 \star}$ and Min-Kai $\operatorname{Lin}^{1,2 \star} \dagger$ \\ ${ }^{1}$ Steward Observatory, University of Arizona, Tucson, AZ 85721, USA \\ ${ }^{2}$ Institute of Astronomy and Astrophysics, Academia Sinica, Taipei 10617, Taiwan
}

Accepted 2016 November 15. Received 2016 November 13; in original form 2016 September 22

\begin{abstract}
The presence of a giant planet in a low-viscosity disc can create a gap edge in the disc's radial density profile sharp enough to excite the Rossby wave instability. This instability may evolve into dust-trapping vortices that might explain the 'banana-shaped' features in recently observed asymmetric transition discs with inner cavities. Previous hydrodynamical simulations of planet-induced vortices have neglected the time-scale of hundreds to thousands of orbits to grow a massive planet to Jupiter size. In this work, we study the effect of a giant planet's runaway growth time-scale on the lifetime and characteristics of the resulting vortex. For two different planet masses ( 1 and 5 Jupiter masses) and two different disc viscosities $(\alpha=$ $3 \times 10^{-4}$ and $3 \times 10^{-5}$ ), we compare the vortices induced by planets with several different growth time-scales between 10 and 4000 planet orbits. In general, we find that slowly-growing planets create significantly weaker vortices with lifetimes and surface densities reduced by more than 50 per cent. For the higher disc viscosity, the longest growth time-scales in our study inhibit vortex formation altogether. Additionally, slowly-growing planets produce vortices that are up to twice as elongated, with azimuthal extents well above $180^{\circ}$ in some cases. These unique, elongated vortices likely create a distinct signature in the dust observations that differentiates them from the more concentrated vortices that correspond to planets with faster growth time-scales. Lastly, we find that the low viscosities necessary for vortex formation likely prevent planets from growing quickly enough to trigger the instability in self-consistent models.
\end{abstract}

Key words: hydrodynamics - instabilities - methods: numerical-planet-disc interactions protoplanetary discs.

\section{INTRODUCTION}

Recent observations by the Atacama Large Millimetre Array (ALMA) have uncovered transition discs with large asymmetries in the dust observed at $\mathrm{mm}$ and sub-mm wavelengths. A variety of mechanisms - including vortices triggered by the Rossby wave instability (RWI) - have been proposed to explain these discs around stars such as Oph IRS 48 (van der Marel et al. 2013), HD 142527 (Casassus et al. 2013; Fukagawa et al. 2013), as well as SAO 206462 and SR 21 (Pérez et al. 2014).

A local minimum or maximum in a disc's radial potential vorticity or 'vortensity' profile can excite the RWI (Lovelace et al. 1999; Li et al. 2000, 2001). Such vortensity extrema often coincide with extrema in the disc's surface density profile, which can arise at dead zone boundaries (e.g. Lyra \& Mac Low 2012; Regály et al. 2012; Miranda et al. 2016) and gap-edges created by giant planets (e.g. Li et al. 2005).

^E-mail: mhammer@as.arizona.edu (MH); kkratter@email.arizona.edu (KMK); mkl@asiaa.sinica.edu.tw (M-KL)

$\dagger$ Steward Theory Fellow.
In both cases, the RWI leads to an exponential growth of nonaxisymmetric modes that develop into a set of vortices in the non-linear regime. In low-mass discs, these vortices will eventually merge to form a single 'banana-shaped' anticyclonic vortex in less than 100 orbits. Single vortices offer a natural explanation for dust asymmetries because they can trap dust particles efficiently in a pressure maximum (e.g. Barge \& Sommeria 1995; Birnstiel, Dullemond \& Pinilla 2013; Lyra \& Lin 2013; Fu et al. 2014b).

Recent observations of the radial gas density profiles interior to the asymmetries have found that some of these regions are depleted, favouring the latter idea that planet-induced vortices are responsible for the asymmetric structures in some transition discs (van der Marel et al. 2016). In this case, a giant planet clears out a gap in the disc, creating sharp peaks in the radial density profile at each gap edge. If the planet is massive enough to steepen a gap edge beyond a critical structure, it will trigger the RWI (Ono et al. 2016). This only happens in discs with low viscosities $\left(\alpha<10^{-3}\right)$. Otherwise, the viscous evolution of the disc will smooth out the gap edge and prevent the instability from arising (e.g. de Val-Borro et al. 2007).

Several groups have conducted numerical simulations of planetinduced vortices to study how well they can explain the recent 
observations of dust asymmetries (Bae, Zhu \& Hartmann 2016) and to explore whether they can survive for long enough to make them observable. In particular, Fu et al. (2014a) used high-resolution twodimensional (2D) simulations to find that a vortex induced by a 5 Jupiter-mass planet can survive for up to 14000 orbits with optimal disc viscosities and temperatures. However, vortex lifetimes can be weakened by a variety of different factors in particular regimes, including self-gravity in very massive discs (Lin \& Papaloizou 2011), three-dimensional structure (Lin 2012; Meheut et al. 2012), and dust feedback from large grains or high concentrations (Fu et al. 2014b). It remains an open question as to how long vortices can survive and whether we should expect them to be observable.

One related question that has been relatively unexplored is the effect of a planet's growth rate on the properties of the vortex. A planet's growth rate can affect the evolution of the vortex it creates because the planet changes the gap structure as it grows. Previous simulations of planet-induced vortices have neglected realistic growth time-scales for the planet, only allowing it to reach its full mass in less than 100 orbits (e.g. de Val-Borro et al. 2007; Fu et al. 2014a; Lobo Gomes et al. 2015; Bae et al. 2016). In this regime, the vortex does not form until after the planet has attained its final mass. With longer growth times, a Jupiter-mass planet will trigger the RWI and induce vortices long before it approaches its final mass (Lin \& Papaloizou 2010), giving the planet and the vortex plenty of time to restructure the gap edge and impact the vortex's further evolution.

Models of core accretion - the mechanism for forming most Jupiter-mass planets - show that a gas giant does not grow to full size within a few dynamical time-scales. The bulk of a planet's growth occurs during the runaway gas accretion phase, when the mass of the planet's gas envelope increases from a few Earth masses to a few hundred Earth masses (Pollack et al. 1996).

Although this phase can be fast compared to the time-scale needed for envelope accretion prior to runaway growth, it is limited by the depleted gas supply in the planet's vicinity once it opens up a gap (e.g. Lissauer et al. 2009). With the disc only gradually supplying the planet with gas, the runaway gas accretion phase can be slowed down to time-scales of hundreds of orbits (D'Angelo \& Lubow 2008), if not thousands in lower viscosity discs (Lissauer et al. 2009). This is one or two orders of magnitude longer than the growth time-scales used in earlier studies of planet-induced vortices.

In this work, we explore the effect of a gap-opening planet's growth time-scale on the lifetimes and other properties of vortices induced by the RWI. We organize this paper as follows: in Section 2, we describe our simulation set-up and quantify our metrics for assessing vortex properties. In Section 3, we present the results from our simulations. In Section 4, we discuss dust concentration in elongated vortices, and put our simulation results in the context of realistic planet formation models. In Section 5, we summarize our results.

\section{METHODS}

\subsection{Numerical methods}

We use FARGO (Masset 2000) to carry out simulations of a gas giant planet with a prescribed growth rate embedded in a low-viscosity disc. FARGO is a 2D finite-difference hydrodynamical code that solves the Navier-Stokes equations in cylindrical polar coordinates $(r, \phi)$. It incorporates the FARGO algorithm, which allows for larger integration timesteps by subtracting out the average azimuthal velocity in each annulus when evaluating the Courant-Friedrich-Levy condition. This technique makes it feasible to carry out a parameter study of simulations with sufficient resolution beyond $10^{4}$ planet orbits.

We prescribe the growth of the planet's mass $m_{\mathrm{p}}$ as a function of time $t$ as

$m_{\mathrm{p}}(t)=M_{\mathrm{p}} \times \begin{cases}\sin ^{2}\left(\pi t / 2 T_{\text {growth }}\right) & t \leq T_{\text {growth }}, \\ 1 & t>T_{\text {growth }},\end{cases}$

where $M_{\mathrm{p}}=m_{\mathrm{p}}\left(T_{\text {growth }}\right)$ is the planet's final mass and $T_{\text {growth }}$ is the planet's growth time-scale, the key parameter in our study.

In principle, one can calculate a 'self-consistent' growth rate based on the amount of material within the planet's Roche radius. However, we instead use the fixed growth prescription above because it allows us to conduct a controlled parameter study of growth time alone. At the resolution of our fiducial simulations, the circumplanetary discs are not sufficiently well resolved to generate realistic growth rates (Zhu 2015). Moreover, accretion on to the planet is a function of a more complex set of disc parameters dependent on thermodynamics and feedback (Ayliffe \& Bate 2009), which we neglect in this study.

To avoid an unphysical build-up of material within the planet's Roche radius, we remove mass from the computational domain according to the prescription from Kley (1999) that is implemented for FARGO's accretion scheme. The total amount of mass removed is relatively small and never exceeds 5 per cent of the planet's mass.

At the radial boundaries, we apply wave killing zones (e.g. de ValBorro et al. 2006) in $r \in[1,1.25] r_{\text {in }}$ and in $r \in[0.84,1] r_{\text {out }}$, where $r_{\text {in }}$ and $r_{\text {out }}$ are the inner and outer edges of the disc, respectively. The outer zone is rapidly damped on a time-scale of 1/500th of the orbital period at the outer boundary so that it approximates a mostly unperturbed region. The inner region is damped at a softer rate of $1 / 3$ rd of the innermost orbital period. Periodic boundaries are applied in the azimuth.

\subsection{Simulations}

We initialize each simulation with a locally isothermal disc that has a power-law radial surface density profile of $\Sigma(r)=\Sigma_{0}\left(r / r_{\mathrm{p}}\right)^{-1}$, where $\Sigma_{0}$ is the initial surface density at the fixed orbital radius of the planet, $r_{\mathrm{p}}$. The value of $\Sigma_{0}$ is set by the total disc mass in the domain, which we fix to $M_{\mathrm{d}}=2 M_{\mathrm{p}}$. We do not include self-gravity because the disc mass is very low with a Toomre $Q>20$ at the location of the vortex in all of our simulations (Toomre 1964). The temperature profile is set to fix the disc aspect ratio $h \equiv H / r=$ 0.06 , where $H$ is the disc's scaleheight. We selected this value of $h$ because Fu et al. (2014a) have shown it maximizes vortex lifetimes.

We simulate an annulus of a disc across a radial domain that extends from $r \in[0.2,5.7] r_{\mathrm{p}}$ and $\phi \in[0,2 \pi]$ in azimuth. This annulus is resolved by $N_{\mathrm{r}} \times N_{\phi}=1024 \times 2048$ arithmetically spaced grid cells in the radial and azimuthal directions, respectively. This grid spacing resolves both the disc's scaleheight $H(r)$ and the planet's Hill radius by at least 11 cells in the outer disc $\left(r>r_{\mathrm{p}}\right)$, our region of interest. Our standard resolution in this region is slightly higher than those used in most other recent studies of planetinduced vortices (e.g. Les \& Lin 2015; Lobo Gomes et al. 2015; Bae et al. 2016), but less than the highest resolution studies ( $\mathrm{Fu}$ et al. 2014a). We carry out convergence tests for select cases at a lower resolution of $768 \times 1536$ and a higher resolution of $1536 \times$ 3072 (see Section 3.4).

In the disc, we place a planet on a fixed circular orbit around a solar mass $\operatorname{star}\left(M_{*}=\mathrm{M}_{\odot}\right)$ with an orbital frequency 
Table 1. Planet growth times for each of the four cases in our simulations.

\begin{tabular}{ccl}
\hline$M_{\mathrm{p}} / M_{\mathrm{J}}$ & $v / r_{\mathrm{p}}^{2} \Omega_{\mathrm{p}}$ & $T_{\text {growth }} / T_{\mathrm{p}}$ \\
\hline 1 & $10^{-6}$ & $10,250,500,1000$ \\
1 & $10^{-7}$ & $10,500,1000,2000$ \\
5 & $10^{-6}$ & $10,500,1000,2000$ \\
5 & $10^{-7}$ & $10,1000,2000,4000$ \\
\hline
\end{tabular}

$\Omega_{\mathrm{p}}=\sqrt{G M_{\odot} / r_{\mathrm{p}}^{3}}$ that corresponds to an orbital period of $T_{\mathrm{p}} \equiv$ $2 \pi / \Omega_{\mathrm{p}}$, where $G$ is the gravitational constant. Our computational units are such that $r_{\mathrm{p}}=\Omega_{\mathrm{p}}=G=M_{*}=1$. The planet's gravitational potential is smoothed out to $0.6 r_{\mathrm{H}}$, where $r_{\mathrm{H}}=(q / 3)^{1 / 3} r_{\mathrm{p}}$ is the planet's Hill radius and $q$ is the planet-to-star mass ratio. In our parameter study, we use two different final planet masses of $q$ $=1 \times 10^{-3}$ and $5 \times 10^{-3}$, which correspond to $M_{\mathrm{p}}=1$ and $5 M_{\mathrm{J}}$ (Jupiter masses) for a solar mass star. We also use two different disc viscosities of $v=10^{-6}$ and $10^{-7}$ in units of $r_{\mathrm{p}}^{2} \Omega_{\mathrm{p}}$, corresponding to $\alpha \approx 3 \times 10^{-4}$ and $3 \times 10^{-5}$, respectively for the standard alpha prescription of $v=\alpha H^{2} \Omega_{\mathrm{p}}$ (Shakura \& Sunyaev 1973). For each of these four combinations of planet masses and disc viscosities, we explore the evolution of the RWI with a focus on the properties and lifetimes of the vortices that form for four different planet growth time-scales (see Table 1).

\subsection{Identifying a vortex signature}

To quantify the vortex properties as a function of the planet's growth time $\left(T_{\text {growth }}\right)$, we need a robust method to identify when they form and dissipate. Quantitative definitions are especially important since vortices do not transition from 'nonexistent' to 'newly formed,' or from 'weak' to 'dead' instantaneously.

We find that the magnitude of the overdensity within the vortex is the best tracer of formation and lifetime. While the planet also produces density enhancements in its spiral wake, these remain fixed in the planet's corotating frame. On the contrary, a vortex orbits at its local Keplerian frequency several Hill radii away from the planet. This difference allows us to differentiate the vortex overdensity from the spiral density waves. We isolate the vortex (with its distinct Keplerian orbital frequency) from the spiral density waves (corotating with the planet) by differencing the surface density fields at successive planet orbits when the planet is at the same location. We find this procedure to be effective in removing the corotational features, leaving only the vortices present in the positive component of the differenced surface density. We then combine all of the positive contributions and define this mass to be the total mass in the vortex, $M_{\text {excess }}$. We use this 'excess mass' to quantitatively determine when a planet triggers the RWI and when a vortex dissipates.

We numerically identify when the RWI is first triggered as the first orbit at which there is a sharp increase in the growth rate of $M_{\text {excess }}$, that is, when it begins to increase roughly exponentially. These times are straightforward to identify from the evolution of the growth rates over time. For a given disc viscosity $v$, we find that these times correspond to a very narrow range of trigger masses across our parameter space. As a result, we do not need a more precise quantitative definition of the trigger point.

While vortices are easy to identify at their peak intensity, pinpointing the orbit at which they disappear is challenging. Vortices can persist at very low mean densities for hundreds of orbits. Thus, we quantify their lifetime based on when $M_{\text {excess }}<0.20 \Sigma_{0} r_{\mathrm{p}}^{2}$ because at this mass, we find that the more concentrated vortices in our study have typically lost $\approx 85-95$ per cent of their peak excess masses (which fall in the range of 1.5-3.5 $\Sigma_{0} r_{\mathrm{p}}^{2}$ ), leaving them with an average overdensity of no more than 10 per cent above the background density. Meanwhile, the choice of a threshold mass does not have a significant effect on the lifetimes of less concentrated vortices since they dissipate more abruptly.

Although it might seem natural to include a quantitative measure of vorticity in our definition of vortex formation and dissipation, we found this to be less reliable. This is because other features at the gap edge also produce spikes in vorticity that make it more difficult to differentiate the vortex's vorticity from that of nearby parts of the disc.

\subsection{Characterizing vortex strength}

We characterize a vortex's strength by quantifying its surface density and azimuthal extent. In general, stronger long-lived vortices have higher surface densities and more concentrated azimuthal extents.

We measure the maximum gas concentration a vortex attains during its lifetime through its peak surface density, $\Sigma_{\text {peak }}$. This is the maximum value of $\Sigma\left(r=r_{\text {vortex }}, \phi=\phi_{\text {peak }}, t\right)$ over time, where $r_{\text {vortex }}$ corresponds to the orbital radius of the vortex defined as the radius at which there is a local maximum in the outer disc $\left(r>r_{\mathrm{p}}\right)$ in the azimuthally averaged surface density profile; $\phi_{\text {peak }}$ is the azimuth at which $\max \left[\Sigma\left(r=r_{\text {vortex }}, \phi\right)\right]$ occurs. We apply a smoothing filter over five orbits before maximizing these values over time. This removes the short time-scale variations, such as those due to the planet's wake. We choose to use the vortex's maximum surface density rather than its average surface density to avoid the difficulty of tracing out the edges of the vortex, which can be ambiguous in many snapshots. None the less, we estimate that the vortex's average surface density is smaller than its maximum surface density by 25 35 per cent.

We estimate a vortex's azimuthal extent, denoted as $\Delta \phi$, by examining $\Sigma\left(r=r_{\text {vortex }}, \phi\right)$ over a wide range of snapshots. We find that a vortex's azimuthal extent is the most concentrated near the time at which its surface density is maximized. For this reason, we characterize a vortex's concentration by the minimum value of $\Delta \phi$ over time, denoted as $[\Delta \phi]_{\min }$. Each concentration is rounded to the nearest $30^{\circ}$, and primarily intended to classify a vortex as 'concentrated' $\left([\Delta \phi]_{\min }<180^{\circ}\right)$, 'elongated' $\left([\Delta \phi]_{\min }>180^{\circ}\right)$, or 'intermediate' $\left([\Delta \phi]_{\min } \approx 180^{\circ}\right)$. Section 4.1 also uses these rough values to show how vortices with different gas azimuthal extents appear in the associated dust observations.

\section{RESULTS}

Our simulations show that planets with longer, more realistic growth times induce vortices with

(i) shorter lifetimes,

(ii) lower peak surface densities, and

(iii) wider azimuthal extents.

We summarize these trends in Fig. 1. We can interpret these results by first considering the gap-opening process, which begins before the RWI is triggered. According to Crida, Morbidelli \& Masset (2006), planets open gaps when

$$
\frac{3}{4} \frac{H\left(r_{\mathrm{p}}\right)}{R_{\mathrm{H}}}+\frac{50}{q \mathrm{Re}} \lesssim 1
$$

where the Reynolds number $\operatorname{Re}=r_{\mathrm{p}}^{2} \Omega_{\mathrm{p}} / v$ is the reciprocal of our dimensionless viscosity. Equation (2) implies gap-opening masses 

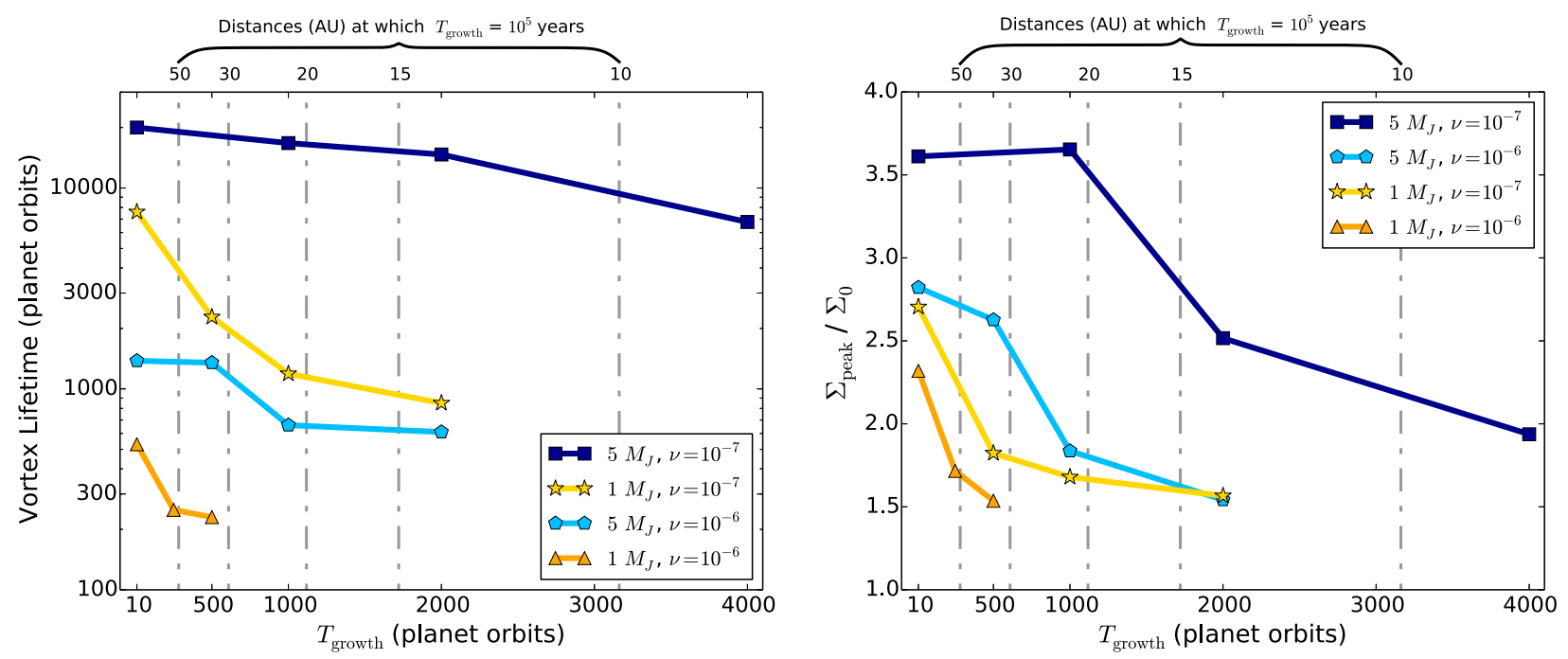

Figure 1. Vortex lifetimes (left-hand panel; defined in Section 2.3) and peak surface densities $\Sigma_{\text {peak }}$ (right-hand panel; defined in Section 2.4) as a function of planet growth time-scale $T_{\text {growth }}$ for each of the four cases in our study. Each dashed vertical line shows the minimum distance at which a growth time-scale in orbits implies a growth time of at least $10^{5}$ years (with $M_{*}=1 \mathrm{M}_{\odot}$ assumed), which serves as a benchmark time-scale from circumplanetary disc accretion rates (see Section 4.3). Left: vortex lifetimes decrease monotonically with larger $T_{\text {growth }}$ in each case. Lower viscosity discs also yield longer vortex lifetimes. Right: $\Sigma_{\text {peak }}$ essentially also decreases monotonically with larger $T_{\text {growth }}$. Higher mass planets induce vortices with higher $\Sigma_{\text {peak }}$. The surface densities are normalized by $\Sigma_{0}$, the initial surface density at the planet's orbital radius, which is also roughly the background density near the vortex.

of $M_{\text {gap }} \approx 0.05 M_{\mathrm{J}}$ and $0.15 M_{\mathrm{J}}$ for $v=10^{-7}$ and $10^{-6}$, respectively. These are well below the final planet masses in our simulations. This suggests that the planet can trigger vortices through the RWI during its growth phase when its mass is still relatively small. This is distinct from previous simulations of planets of at least Jupiter mass where the planet reaches its full mass almost immediately after vortices form (e.g. de Val-Borro et al. 2007; Fu et al. 2014a).

Indeed, using $M_{\text {excess }}$ shown in Fig. 2 - our measure of vortex presence - we find that the first spike in $M_{\text {excess }}$ occurs when $m_{\mathrm{p}}$ $\approx 0.03-0.06 M_{\mathrm{J}}$ for $v=10^{-7}$, and at roughly $m_{\mathrm{p}} \approx 0.2 M_{\mathrm{J}}$ for $v=10^{-6}$, both independent of $T_{\text {growth }}$. These values of $m_{\mathrm{p}}$ are close to the gap-opening masses $M_{\text {gap }}$ estimated above, suggesting that the RWI develops soon after gap-opening begins. Once the RWI is triggered, $M_{\text {excess }}$ enters a short exponential growth phase lasting for $50-100$ orbits. We find that this time-scale, $T_{\text {exp }}$, is also approximately the time it takes for the initial vortices to merge into a single vortex (azimuthal wavenumber $m=1$ ), and that $T_{\exp }$ does not vary significantly with $T_{\text {growth }}$.

Despite similar trigger masses and exponential growth phases, the resulting vortices are in fact quite different when the planet takes longer to grow. These differences arise because once the vortex emerges, it begins to back-react on the gap edge, smoothing it out. The growing planet competes against the already-present vortex to sharpen the gap edge. Varnière, Quillen \& Frank (2004) show that the ratio of the surface density at the gap centre, $\Sigma_{\text {gap }}$ to that at the gap edge, $\Sigma_{\text {edge }}$, depends on planet mass and Reynolds numbers as:

$\frac{\Sigma_{\text {edge }}}{\Sigma_{\text {gap }}} \propto q^{2} \operatorname{Re}$

For a growing planet, we expect the steepness of the gap edge to evolve on a time-scale of order $T_{\text {gap }} \equiv q(t) / \dot{q}(t)$.

In Fig. 3, we show that the azimuthal extents of the vortices are well divided in a parameter space $q^{2} \operatorname{Re}$ versus $<\dot{q}>^{-1}$, where angle brackets represent a time average. Specifically, the properties of the vortex depend on both the steepness of the gap edge and how quickly the gap edge evolves. This dependence reflects the competition between $T_{\text {gap }}$, the time-scale for gap sculpting; and $T_{\text {exp }}$, the roughly constant time-scale which governs how quickly the vortex can back-react on the gap. To verify the robustness of our choice of dimensionless numbers to map vortex properties, we ran short-term simulations with different combinations of $q$ and $v$ that more densely sample $q^{2} \mathrm{Re}$, and overlap with values already in our full length parameter study. We find excellent consistency between vortex azimuthal extents in the overlapping cases. When $T_{\text {gap }}>T_{\exp }$ (that is, for slow planet growth), the vortex begins to smooth out the gap edge while the planet is still at a low mass, preventing the gap edge from attaining the maximum steepness for a given pair of $q$ and Re. This leads to the vortex having a smaller saturation amplitude in density and a less concentrated azimuthal extent. When $T_{\text {gap }} \lesssim$ $T_{\text {exp }}$, the planet grows quickly, continually steepening the gap edge. Thus, the back-reaction from the vortex only becomes important when the planet is more massive and the gap edge sharper. This leads to the vortices becoming stronger and more concentrated.

For more massive planets with larger $q^{2} \mathrm{Re}$, we observe that the azimuthal extent of a vortex can evolve from 'elongated' to 'intermediate'. This behaviour arises because the planet continues to steepen the gap edge long after the exponential growth phase of $M_{\text {excess. }}$ As a result, the slope of the dividing line in Fig. 3 is positive. We emphasize that the largest distinction between the vortices is their azimuthal extents, rather than their peak mass or saturation amplitude. This is consistent with the linear scaling between the RWI growth rate and saturation amplitude found by Meheut, Lovelace \& Lai (2013).

With this interpretation in hand, we now provide more detailed descriptions of the different cases. We describe the vortices induced by $5 M_{\mathrm{J}}$ planets in Section 3.1 and the vortices in induced by $1 M_{\mathrm{J}}$ planets in Section 3.2. We observe and explain the repeated vortex formation in Section 3.3. Lastly, we show that the 

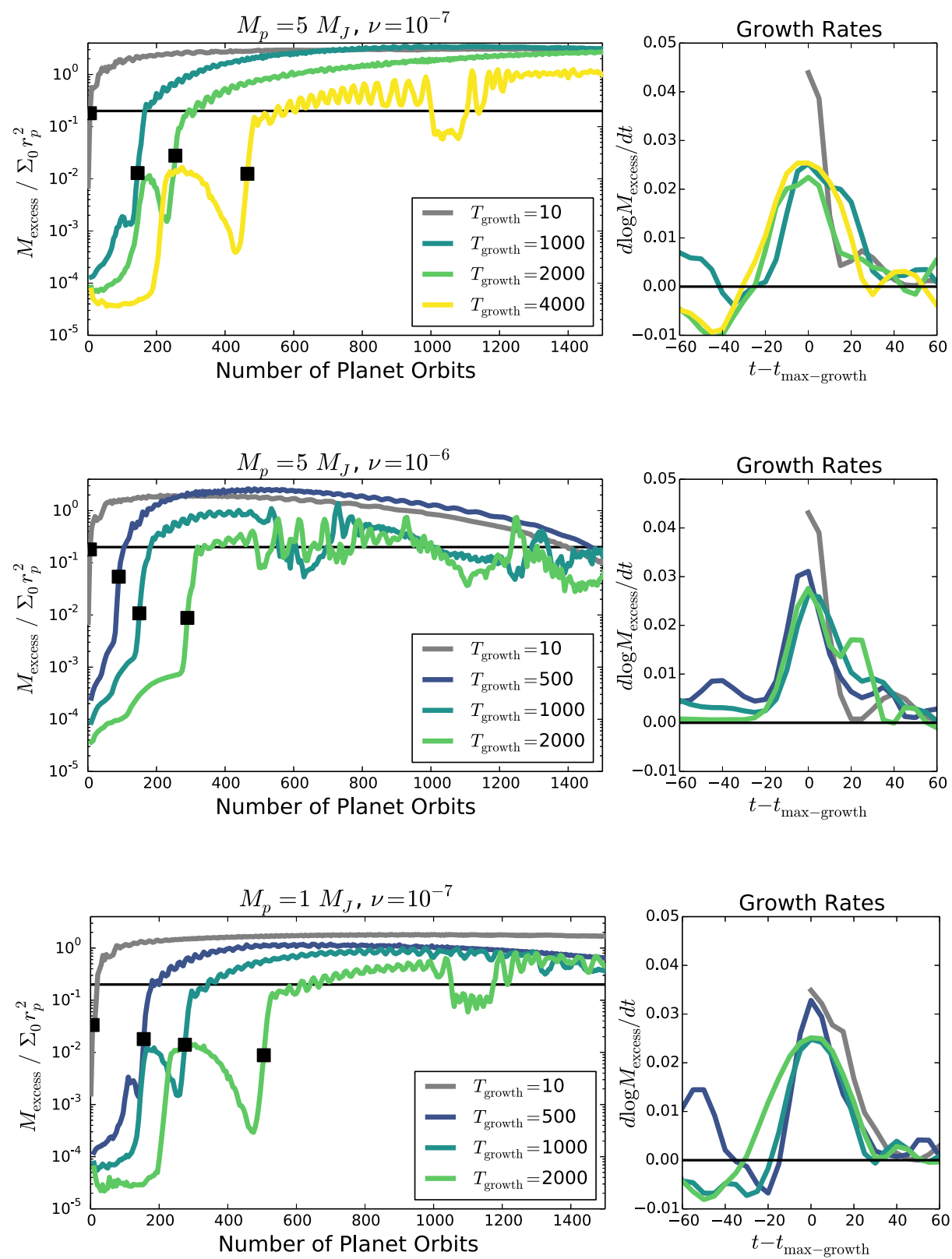

Figure 2. Left-hand panels: the excess mass, $M_{\text {excess }}$, in a vortex over time across our parameter space. When $M_{\text {excess }}>0.2 \Sigma_{0} r_{\mathrm{p}}^{2}$ (marked with a horizontal line), a vortex is alive. Right-hand panels: exponential growth rates across our parameter space, during the early growth stages of each vortex and centred at the snapshot with the maximum growth rate (denoted in the left-hand panels with black squares). Regardless of $m_{\mathrm{p}}$ and $\nu$, all of the slowly-grown planets with $T_{\text {growth }} \gg 10 T_{\mathrm{p}}$ trigger vortices with similar maximum growth rates of $\mathrm{d} \log M_{\text {excess }} / \mathrm{d} t \approx 0.025$. After reaching this point, the growth rates immediately begin to decline.

underlying trends in our results converge at different resolutions in Section 3.4 .

\subsection{Vortices induced by higher mass planets $\left(M_{\mathrm{p}}=5 M_{\mathrm{J}}\right)$}

We find that for $T_{\text {growth }}=10 T_{\mathrm{p}}$, planets with $M_{\mathrm{p}}=5 M_{\mathrm{J}}$ in discs with a low viscosity $\left(v=10^{-7}\right)$ generate strong, concentrated vortices that survive well beyond $10^{4}$ orbits, in agreement with previous studies (Fu et al. 2014a). Unlike in any of the other cases (see below), planets with $T_{\text {growth }}=2000 T_{\mathrm{p}}$ also generate rather strong vortices with lifetimes just above $10^{4} T_{\mathrm{p}}$ and $\Sigma_{\text {peak }}>2.5 \Sigma_{0}$. Nevertheless, these vortices are still weaker than those produced by a rapidly grown planet. We find that it takes increasing $T_{\text {growth }}$ to $4000 T_{\mathrm{p}}$ to reduce the vortex's $\Sigma_{\text {peak }}$ below $2 \Sigma_{0}$ (see Fig. 4 ). These vortices still survive more than 7000 orbits with azimuthal extents that are relatively concentrated $\left([\Delta \phi]_{\min } \approx 180^{\circ}\right)$ rather than elongated.

In a high-viscosity disc $\left(v=10^{-6}\right)$, a $5 M_{\mathrm{J}}$ planet with $T_{\text {growth }}=$ $500 T_{\mathrm{p}}$ induces a vortex that survives just as long $(\approx 1300$ orbits $)$ and has a similar surface density $\left(\Sigma_{\text {peak }} \approx 2.7 \Sigma_{0}\right)$ as a vortex generated by a rapidly grown planet. However, with $T_{\text {growth }}=1000 T_{\mathrm{p}}$, the vortex's lifetime is halved, its azimuthal extent doubles (from $[\Delta \phi]_{\min }$ $=90^{\circ}$ to $180^{\circ}$ ), and $\Sigma_{\text {peak }}<2 \Sigma_{0}$. Fig. 5 shows snapshots of this case compared to the rapid growth case. With $T_{\text {growth }}=2000 T_{\mathrm{p}}$, 


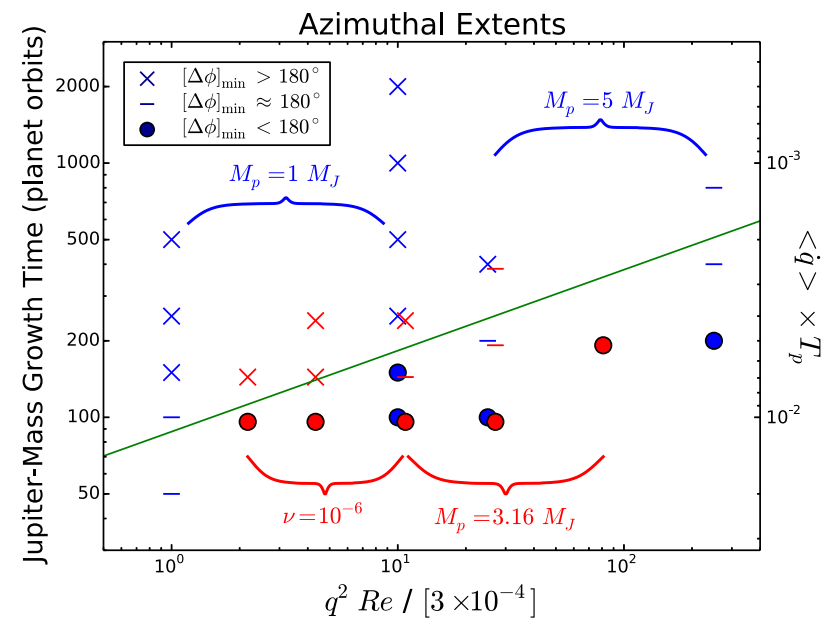

Figure 3. Vortex shapes across our parameter space (blue points are part of our main study; red points are added). The green line shows that the two parameters on the axes related to gap steepening separate elongated vortices (above) from concentrated ones (below). We determine the parameters for this dividing line by assuming a power-law parametrization of $<\dot{q} / 10^{-3}>^{-1}=A \times\left(q^{2} \mathrm{Re}\right)^{B}$. We then use a soft-margin support vector machine model with $c=25$ (implemented by SVM-Light, Joachims 1999) to determine that the values of $A=88$ and $B=0.32$ create the maximum margin between the set of elongated vortices and the set of concentrated vortices. even though the RWI is triggered with high- $m$ azimuthal modes, we do not observe them to evolve and merge into a clear $m=1$ vortex.

\subsection{Vortices induced by lower mass planets $\left(m_{\mathrm{p}}=1 M_{\mathrm{J}}\right)$}

In the rapid growth case where $T_{\text {growth }}=10 T_{\mathrm{p}}$, we find that Jupitermass planets in discs with a low viscosity $\left(v=10^{-7}\right)$ also induce strong, concentrated vortices. These vortices have relatively long lifetimes of several thousand orbits, which is consistent with previous studies (e.g. Les \& Lin 2015; Lobo Gomes et al. 2015) and only about a factor of $\sim 3$ shorter than those with the higher mass planet. However for $T_{\text {growth }} \geq 500 T_{\mathrm{p}}$, lifetimes decrease by an order of magnitude, azimuthal extents double ([ $\Delta \phi]_{\min } \approx 240^{\circ}$ compared to $120^{\circ}$ ) and $\Sigma_{\text {peak }}$ drops very quickly to $<2 \Sigma_{0}$ (see Fig. 1). Fig. 6 shows surface density snapshots that highlight the morphological differences between these concentrated and elongated vortices over the course of their lifetimes.

While slow growth does not suppress vortex formation entirely in low-viscosity discs, we find that in the higher viscosity disc with $v=10^{-6}$, growth times over 1000 orbits inhibit vortex formation completely for $M_{\mathrm{p}}=1 M_{\mathrm{J}}$. With $T_{\text {growth }}$ of 250 and $500 T_{\mathrm{p}}$, planets create vortices that are a little more elongated $\left([\Delta \phi]_{\min } \approx 240^{\circ}\right.$ compared to $180^{\circ}$ ), have $\Sigma_{\text {peak }}<2 \Sigma_{0}$, and shorter lifetimes by $\sim 50$ per cent compared to the rapid growth case. In these slowlygrown cases, about half of the reported vortex lifetime transpires before the high- $m$ RWI modes have merged into a single vortex. For
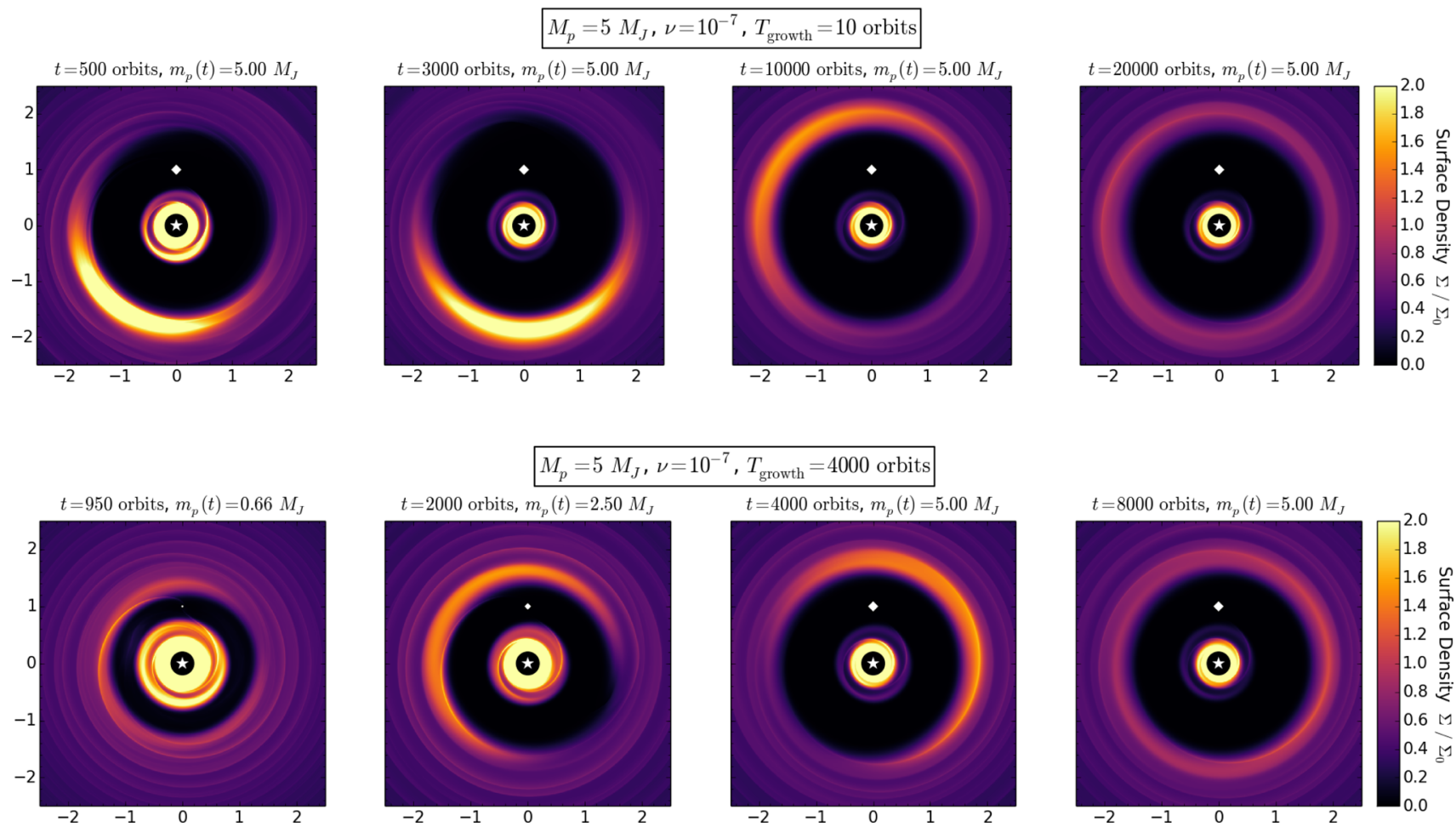

Figure 4. Surface density snapshots for $M_{\mathrm{p}}=5 M_{\mathrm{J}}$ and $v=10^{-7}$, for $T_{\text {growth }}=10,2000$, and $4000 T_{\mathrm{p}}$. The title of each snapshot indicates the time, and the mass of the planet at that time. The planet is indicated by a diamond above the star in each snapshot, and its size is scaled relative to the planet's final mass. Top: with $T_{\text {growth }}=10 T_{\mathrm{p}}$, a strong vortex forms very quickly and persists at peak strength through 3000 orbits. After 10000 orbits, the vortex has weakened and spreads out slightly in azimuth, but is still rather prominent. It does not die until 20000 orbits. Despite starting out weaker, the vortices with $T_{\text {growth }}=$ 1000 and $2000 T_{\mathrm{p}}$ (not shown) also remain prominent after $10^{4}$ orbits, just like in the rapid growth case, but ultimately dissipate earlier. Bottom: with $T_{\text {growth }}=$ $4000 T_{\mathrm{p}}$, the vortex's peak density reaches only about half that of the rapid growth case, and never becomes more concentrated than $180^{\circ}$. In-between the first two snapshots shown, the vortex dies at about 1000 orbits and reforms $\sim 50$ orbits later. After the planet finishes growing at 4000 orbits, the vortex is only as strong as the vortices near 10000 orbits for the other growth time-scales (compare the third column of panels). The vortex then fades faster, dying out within 8000 orbits. 

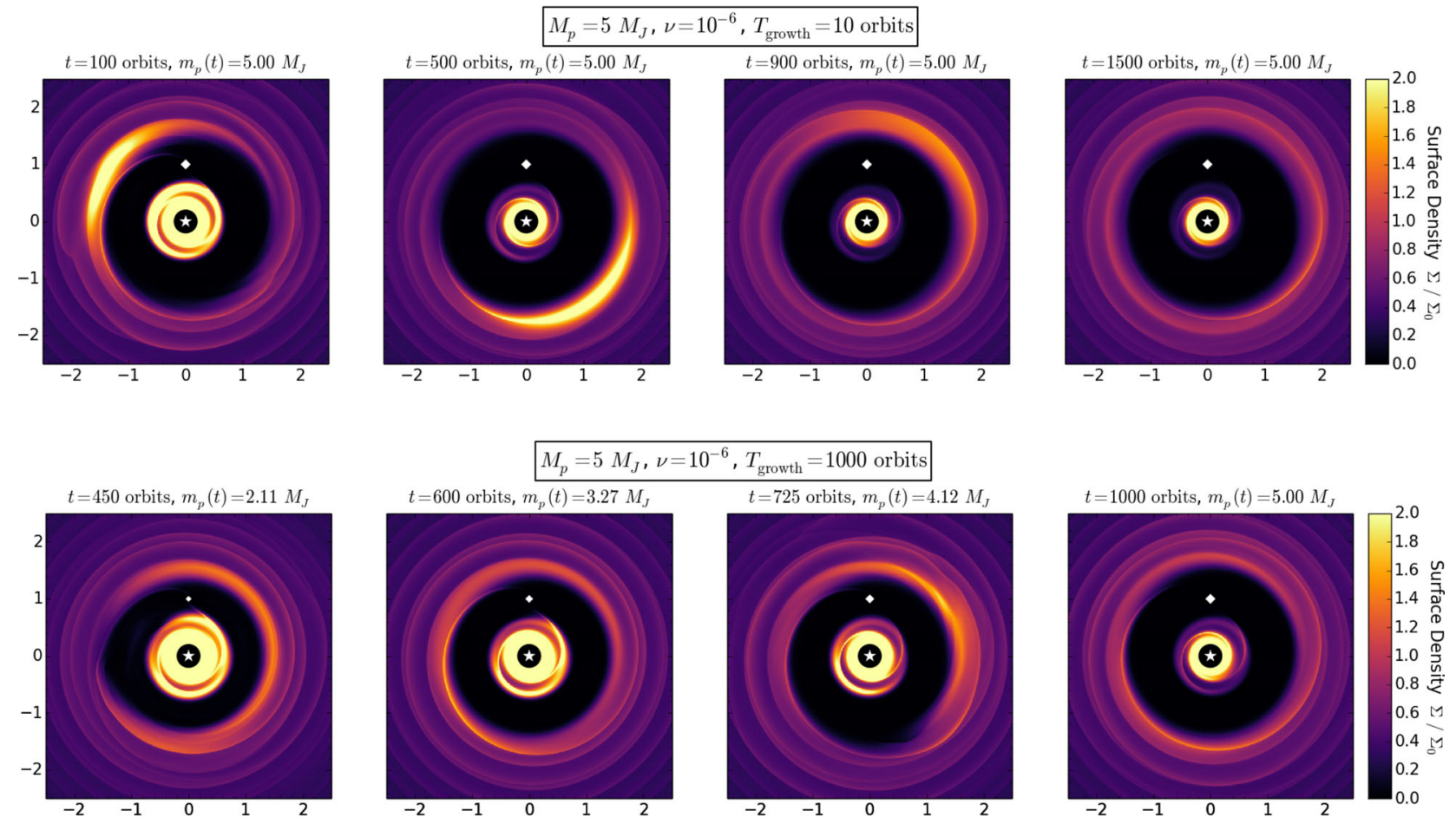

Figure 5. Surface density snapshots for $M_{\mathrm{p}}=5 M_{\mathrm{J}}$ and $v=10^{-6}$, for $T_{\text {growth }}=10,500$, and $1000 T_{\mathrm{p}}$. See Fig. 4 for more details. Top: with $T_{\text {growth }}=10 T_{\mathrm{p}}$, a strong vortex forms by 100 orbits and remains near peak strength at 500 orbits. By 900 orbits, the vortex has quickly faded and by 1500 orbits, it has died out. The vortex with $T_{\text {growth }}=500 T_{\mathrm{p}}$ (not shown) develops similar properties to the vortex in this rapid growth case, despite forming slightly later. Bottom: with $T_{\text {growth }}=1000 T_{\mathrm{p}}$, a much weaker, more elongated vortex initially forms. It dies out by 600 orbits. As the planet continues to grow, it then re-triggers a vortex of similar strength about 100 orbits later. This vortex then dies out near 1000 orbits when the planet has finished growing. The longest time-scale $T_{\text {growth }}=$ $2000 T_{\mathrm{p}}$ (not shown) never produces a clear $m=1$ vortex.

this reason along with their shorter lifetimes, vortices generated by a Jupiter-mass planet in a high-viscosity disc with $v=10^{-6}$ are not well suited to explain observed disc asymmetries.

\subsection{Vortex reformation}

In a few cases, we observe a distinct behaviour with large values of $T_{\text {growth }}$ (slow growth times). When a vortex is initially weak, it can fade into a ring before the planet reaches its final mass. Since the planet continues to clear out the gap as it grows, these rings can develop sufficiently steep edges to re-trigger the RWI and form a new vortex. This only occurs for weak vortices, which can fade quickly before the growth phase ends and do not smooth out the nearby gap edge as much as stronger vortices formed by quickly accreting planets. Note that secondary vortices have also been observed by Lobo Gomes et al. (2015) for discs with a nonisothermal equation of state, albeit at a different location further from the planet.

The bottom panel of Fig. 6 shows an example of a vortex reforming in a low-viscosity disc $\left(v=10^{-7}\right)$ for a $1 M_{\mathrm{J}}$ planet with $T_{\text {growth }}$ $=2000 T_{\mathrm{p}}$. The vortex initially forms and dies out after several hundred orbits. Shortly after, it reforms at a similar level of strength in terms of $\Sigma_{\text {peak }}$ and $[\Delta \phi]_{\text {min }}$, and also survives for several hundred orbits.

Along the same lines, we find the three cases of (A) $M_{\mathrm{p}}=5 M_{\mathrm{J}}$, $v=10^{-7}, T_{\text {growth }}=4000 T_{\mathrm{p}}$; (B) $M_{\mathrm{p}}=5 M_{\mathrm{J}}, v=10^{-6}, T_{\text {growth }}=$ $1000 T_{\mathrm{p}}$; and $(\mathrm{C}) M_{\mathrm{p}}=1 M_{\mathrm{J}}, v=10^{-6}, T_{\text {growth }}=500 T_{\mathrm{p}}$ also create vortices that die out and reform while the planet is still growing. The first two cases are shown in the bottom panels of Figs 4 and 5 .
The first case is unique because the first-generation vortex dies out very early on in the planet's growth phase. This allows the secondgeneration vortex to become much stronger than the first one.

We cannot determine if the stronger, concentrated vortices in our study can reform, since they die out long after the planet has grown to its full mass. However, all of the weaker vortices that reform begin their second-generation phase at a level of strength near the strength of the first-generation vortex shortly before it dissipated. Therefore, even if the strongest vortices can reform, we do not expect them to reach the densities or lifetimes of the first-generation vortices unless the planet has a significant fraction of its growth phase remaining.

\subsection{Convergence tests}

The standard resolution in our parameter study resolves the disc rather well in the vicinity of the planet and the vortex compared to other studies of planet-induced vortices (e.g. Les \& Lin 2015; Lobo Gomes et al. 2015; Bae et al. 2016). None the less, computational planet-disc interaction studies are notoriously susceptible to resolution artefacts (e.g. de Val-Borro et al. 2007; Muñoz et al. 2014).

To confirm the above trends that we observe, we ran convergence tests for several cases: (1) $M_{\mathrm{p}}=5 M_{\mathrm{J}}, v=10^{-6}$, with $T_{\text {growth }}=$ 10,500 , and $1000 T_{\mathrm{p}}$; and (2) $M_{\mathrm{p}}=1 M_{J}, v=10^{-7}$, with $T_{\text {growth }}$ $=500$ and $1000 T_{\mathrm{p}}$. We find that the trend that increasing $T_{\text {growth }}$ produces increasingly weaker vortices is upheld at each resolution. Therefore, we are confident that this trend is physical.

The tests for the $5 M_{\mathrm{J}}$ planets converge well quantitatively, producing vortex lifetimes and $\Sigma_{\text {peak }}$ consistent with the standard resolution simulations to within 15 percent. For $1 M_{\mathrm{J}}$ planets, the 

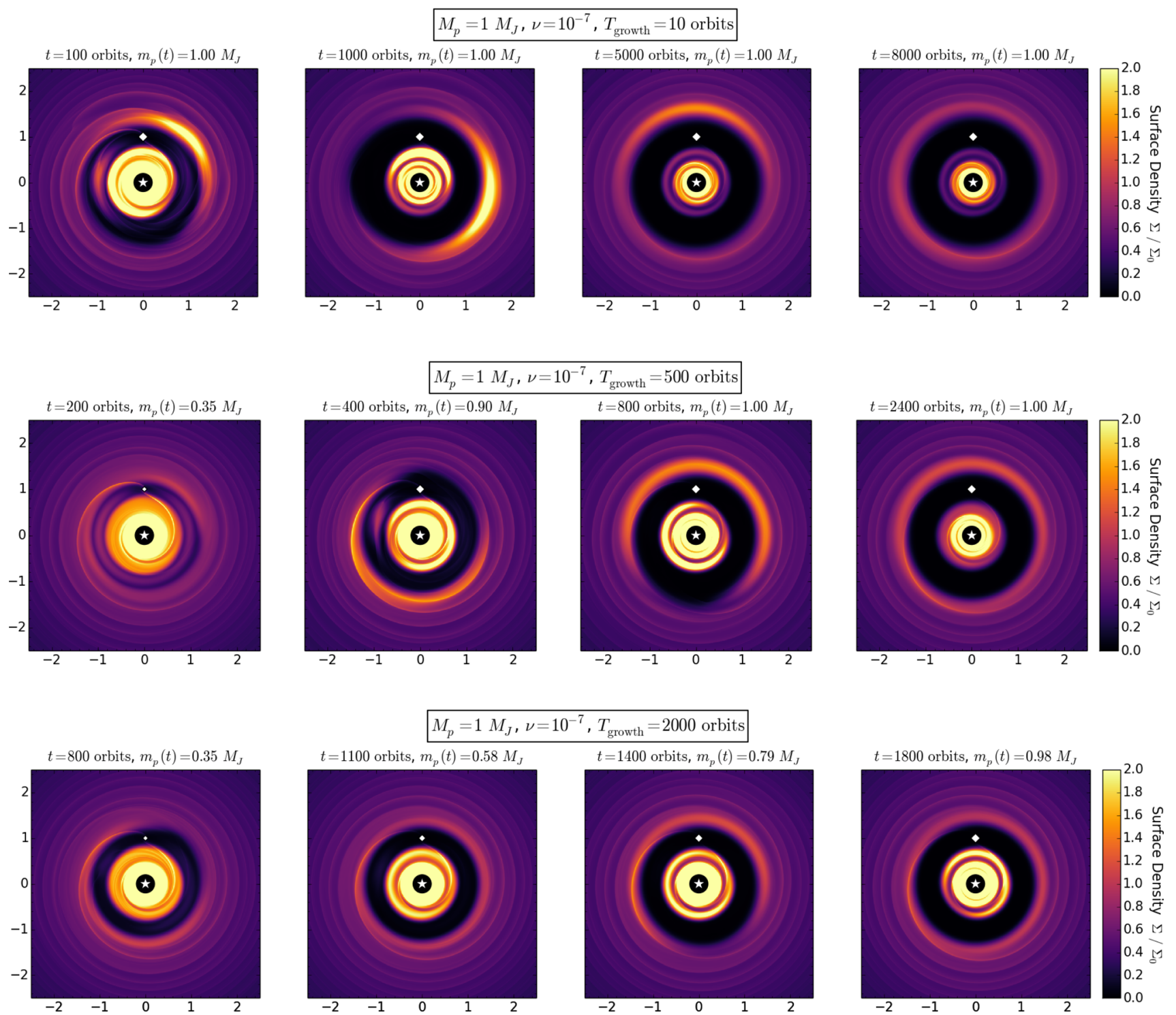

Figure 6. Surface density snapshots for $M_{\mathrm{p}}=5 M_{\mathrm{J}}$ and $v=10^{-7}$, for $T_{\text {growth }}=10,500$, and $2000 T_{\mathrm{p}}$. See Fig. 4 for more details. Top: with $T_{\text {growth }}=10 T_{\mathrm{p}}$, a strong vortex forms by 100 orbits, and survives for several thousand orbits. Middle: with $T_{\text {growth }}=500 T_{\mathrm{p}}$, the planet triggers the instability after 200 orbits, but the resulting $m=1$ vortex is much more elongated with $[\Delta \phi]_{\min } \approx 240^{\circ}$. Bottom: with $T_{\text {growth }}=2000 T_{\mathrm{p}}$, although a vortex forms by 800 orbits, it fades into a ring by 1100 orbits. Then, while the planet is still growing, it re-triggers a vortex at 1400 orbits. This new vortex has roughly the same density as the earlier one and like the first, it also quickly dies into a ring just a few hundred orbits later.

relation between $T_{\text {growth }}$ and vortex lifetime remains, but the lifetimes vary with resolution. Specifically, the lifetimes for all cases decrease with increasing resolution, suggesting that the estimated lifetimes we present in Fig. 1 as well as in Section 4.3 may be upper limits. We suspect that the difference in behaviour as a function of mass is related to the better resolution of the planet's Hill radius and spiral shocks from the planets in the higher mass case, as vortex dissipation may be related to its interactions with these planetary shocks (Fu et al. 2014a).

\section{OBSERVATIONAL IMPLICATIONS}

The disc asymmetries attributed to vortices are observed in $\mathrm{mm}-$ $\mathrm{cm}$ dust grains (e.g. van der Marel et al. 2013). Although the gas overdensity may be modest, a vortex acts as a very efficient dust trap due to the local pressure maxima at the centre. To quantify the impact of planet growth time on the properties of observed vortices, we must model the distribution of dust particles in a range of sizes within our simulated vortices. We have shown that $T_{\text {growth }}$ dramatically alters the concentration and azimuthal extent of planetinduced vortices. To illustrate the differences between concentrated and elongated vortices in dust observations, we make use of the vortex dust-trapping model developed by Lyra \& Lin (2013).

\subsection{Dust-trapping model}

We approximate our simulated vortices as Kida vortices with elliptical streamlines and density contours of constant aspect ratio $\chi$ (Kida 1981). We estimate the vortex aspect ratio as

$\chi=r \Delta \phi / \Delta r$ 


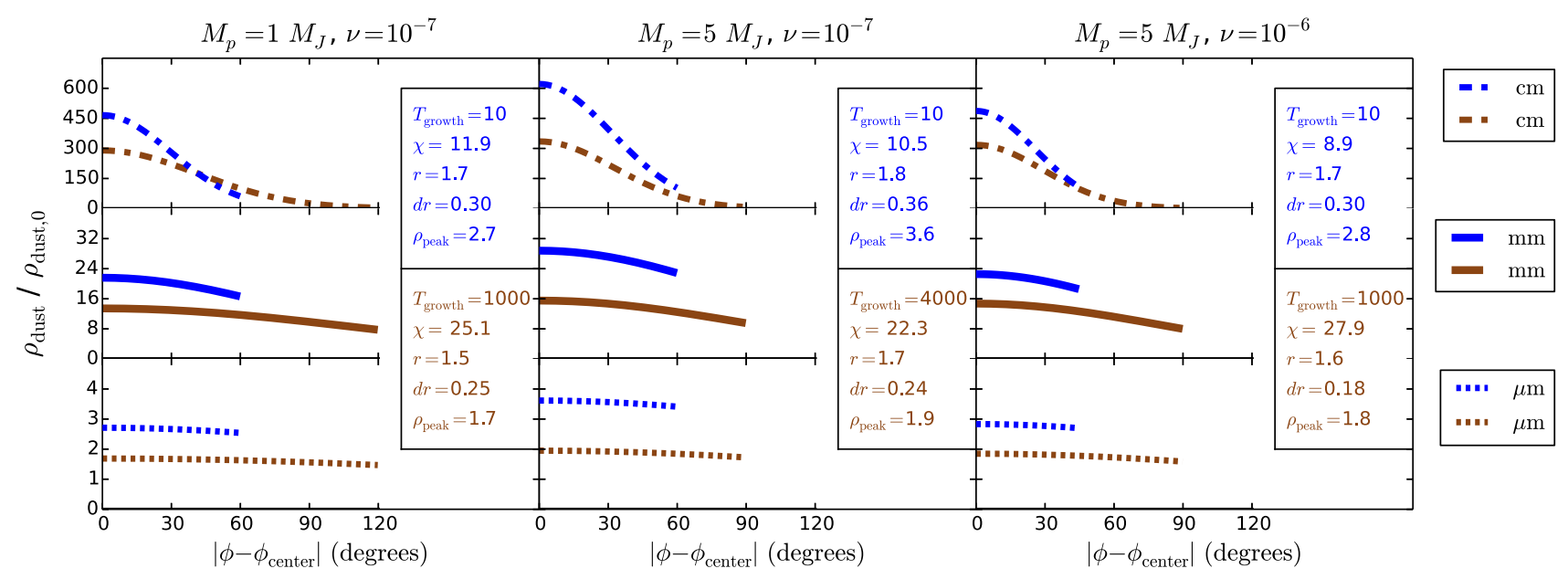

Figure 7. Comparison of the dust distributions ( $\mathrm{cm}$ size, $\mathrm{mm}$ size, and $\mu \mathrm{m}$ size) produced by vortices in three of the rapid growth cases (blue lines; parameters listed in the top windows) to those from a longer growth time-scale (brown lines; parameters listed in the bottom windows). Distributions are calculated using equation (5). The gas azimuthal extents can be extracted from these azimuthal dust density distributions through the dust extent of the mm-size or $\mu \mathrm{m}$-size particles, or the steepness of the distribution of $\mathrm{cm}$-size particles. The distributions are normalized to the initial dust density at the location of the planet.

where $r \Delta \phi$ and $\Delta r$ are the vortex azimuthal and radial extents, respectively, that are measured from our simulations. The azimuthal extent is chosen to be $[\Delta \phi]_{\min }$ and the radial extent is estimated from the differenced surface density.

We then apply Lyra and Lin's model for the steady-state dust distribution in a disc vortex:

$\rho_{\text {dust }}(a)=\epsilon \rho_{\text {peak }}(S+1)^{3 / 2} \exp \left\{-\frac{a^{2} f^{2}(\chi)}{2 H^{2}}(S+1)\right\}$

[see their equation (64)], where $a$ is the semiminor axis of an elliptical density contour inside the vortex; $\rho_{\text {peak }}$ is the peak gas density, here inferred from our simulations (see Fig. 1); $S$ is the ratio of the particles' Stokes number to the dimensionless diffusion coefficient in the vortex; $f(\chi)$ is a scale function of order unity [see their equation (35)]; and $\epsilon$ is the dust-to-gas ratio. We fix $S$ to be $3 \times 10^{-3}, 3$, and 30 to represent $\mu \mathrm{m}$-size, mm-size, and $\mathrm{cm}$-size particles, respectively. The value of $S$ can vary by about an order of magnitude for each size for reasonable choices of the diffusion coefficient.

\subsection{Dust distributions in concentrated and elongated vortices}

Fig. 7 compares the $\mu \mathrm{m}$-size, mm-size, and cm-size dust distributions of concentrated and elongated vortices for three different combinations of planet masses and disc viscosities.

In general, we find that the distributions of mm-size particles are the best tracer for the underlying gas vortex's azimuthal extent since they are effectively concentrated and maintain a fairly constant overdensity from the vortex centre to the outer edge. The distributions of $\mu \mathrm{m}$-size particles are also relatively flat throughout the vortex, but they are not trapped as efficiently and thus have a lower density enhancement throughout.

With larger particles ( $\mathrm{cm}$ size), the dust extents cannot be used to measure the gas extents directly since the dust concentrations approach zero inside the outer edge of the vortex. Instead, we find that the slopes of these steeper azimuthal distributions are a better indicator of the gas azimuthal extent. However, the differences in these slopes for vortices with different azimuthal extents can be very small ( $<10$ per cent) when the distributions are normalized to the density at the vortex centre, making the extents difficult to measure precisely with this size particle.

This simplified model illustrates that two types of vortices might be distinguishable at mm wavelengths. Since $\mu \mathrm{m}$-size particles are also good at tracing the azimuthal extents, any contamination to $\mathrm{mm}$ observations from smaller wavelengths should only help distinguish an elongated vortex from a concentrated vortex. We expect that the dust overdensities shown in Fig. 7 are likely underestimates of the observable asymmetry, as we have not accounted for the removal of dust elsewhere along the azimuth at the radial location of the vortex. However, the simulated vortices have more complicated streamline and density structures at their outer edges, which might make dust trapping less effective. A more detailed dust-trapping model that is more appropriate for elongated vortices is needed to better assess the viability of measuring a vortex's azimuthal extent for constraining the associated planet's growth time-scale.

\subsection{Constraints from planet formation models}

We have shown that planets must form within a few thousand orbits or less in order to induce long-lived vortices. We now compare these time-scales with realistic growth rates from planet formation models, finding that extreme conditions are required to satisfy the growth rate constraint.

First, we consider the fastest possible accretion rates. A strong upper limit on the planet's growth is set by allowing the planet to efficiently accrete from its feeding zone in the 2D limit as

$\dot{M}_{\mathrm{p}} \approx \Sigma \Omega_{\mathrm{p}} R_{\mathrm{H}}^{2}$

(Youdin \& Kenyon 2013). To evaluate this rate, we assume a surface density profile that is proportional to the Minimum Mass Solar Nebula (MMSN),

$\Sigma(r)=2200 F\left(\frac{r}{1 \mathrm{au}}\right)^{-3 / 2} \mathrm{~g} \mathrm{~cm}^{-2}$,

where $F$ is an enhancement factor ( $F=1$ refers to the MMSN). With this profile, the maximum growth rate of the planet is

$\dot{M}_{\mathrm{p}} \approx 1.1 \times 10^{-2} F\left(\frac{r}{5 \mathrm{au}}\right)^{1 / 2}\left(\frac{q}{5 \times 10^{-4}}\right)^{2 / 3} \frac{M_{\mathrm{J}}}{T_{\mathrm{p}}}$, 
where we have used the value of $q=0.5 M_{\mathrm{J}} / \mathrm{M}_{\odot}$ for the Hill radius scaling as the planet cannot initially accrete from the Hill radius corresponding to its final mass. This upper limit corresponds to $T_{\text {growth }}=M_{\mathrm{p}} / \dot{M}_{\mathrm{p}} \approx 90 T_{\mathrm{p}}$ for a Jupiter-mass planet that forms at 5 au in the MMSN. This time-scale is safely an order of magnitude shorter than the critical growth rate required for vortex formation in our models $\left(T_{\mathrm{p}} \sim 1000 T_{\mathrm{p}}\right)$. Thus, if planets were able to accrete at this maximal rate, vortex formation would be robust. However, achieving these maximum growth rates is unlikely when a planet has already opened up a gap, which occurs at a relatively low mass in a low-viscosity disc.

A second simple estimate for planet growth time can be derived from considering the background accretion rate through the disc. In steady state, we expect the disc to funnel material into the vicinity of the planet at the disc accretion rate $\dot{M}_{\mathrm{d}}=3 \pi v \Sigma$. Since we expect $\dot{M}_{\mathrm{p}} \lesssim \dot{M}_{\mathrm{d}}$ [Lubow \& D'Angelo (2006) find that $\dot{M}_{\mathrm{p}}$ never exceeds 80 per cent of $\dot{M}_{d}$ ], for low-viscosity discs the background accretion rate sets a more stringent limit on the planet growth times than the estimate above. Equating the disc accretion rate to the planet growth rate, we define the critical surface density for vortex formation to be

$\Sigma_{\text {crit }} \equiv \frac{\dot{M}_{\mathrm{d}}}{3 \pi v}=\frac{M_{p}}{T_{\text {growth, }}} \frac{1}{3 \pi v}$,

where $T_{\text {growth,c }}$ is the critical planet growth timescale that still allows vortices to form (which is a function of $M_{\mathrm{p}}$ and $v$ ). As above, we scale $\Sigma_{\text {crit }}$ to the MMSN to obtain $F_{\text {crit }}$, the enhancement over the MMSN required to allow sufficiently rapid accretion to induce vortex formation. We find

$F_{\text {crit }}=29.1\left(\frac{M_{\mathrm{p}}}{1 M_{\mathrm{J}}}\right)\left(\frac{1000 T_{\mathrm{p}}}{T_{\text {growth, }}}\right)\left(\frac{r}{5 \mathrm{au}}\right)^{-1 / 2}\left(\frac{10^{-6} r_{\mathrm{p}}^{2} \Omega}{v}\right)$.

Aside from the discrepancy with observed disc masses (Andrews et al. 2011), this very high surface density is incompatible with the assumption of a low-disc viscosity, because it corresponds to a disc with a small Toomre $Q$. For the passive irradiated disc profile from Chiang \& Goldreich (1997) [see their equation (14a)] with

$T=T_{\text {mid }}\left(\frac{r}{5 \mathrm{au}}\right)^{-3 / 7}$,

where $T_{\text {mid }}$ is midplane temperature at 5 au in the fiducial T-Tauri irradiation model,

$Q \approx 0.77\left(\frac{r}{5 \mathrm{au}}\right)^{-\frac{3}{14}}\left(\frac{M_{*}}{\mathrm{M}_{\odot}}\right)^{1 / 2}\left(\frac{T_{\mathrm{mid}}}{75 \mathrm{~K}}\right)^{1 / 2}\left(\frac{29.1}{F_{\text {crit }}}\right)$.

Discs with $Q<2$ are subject to gravitational instabilities that generate effective $\alpha>0.01$ or $v>10^{-4} r_{\mathrm{p}}^{2} \Omega_{\mathrm{p}}$ (Kratter \& Lodato 2016). Even for a factor of $\sim 5-10$ decrease in $F_{\text {crit }}$ (increase in $Q$ ), disc self-gravity may suppress large-scale vortex formation via the RWI (Lin \& Papaloizou 2011). We conclude that the planet growth rates required for vortex formation are incompatible with growth via mass re-supply from the disc.

Neither of the above estimates captures the details of planetary accretion. More realistic simulations of Jupiter's formation that account for gap opening and feedback produce accretion rates in line with our second estimate, which is 1-2 orders of magnitude slower than the rates needed for robust vortex formation. Lissauer et al. (2009) model the growth of a $1 M_{\mathrm{J}}$ planet at 5 au in a disc with $v=10^{-6} r_{\mathrm{p}} \Omega_{\mathrm{p}}^{2}$ and $F \approx 3.5$. They find that the runaway gas accretion phase lasts for $4 \times 10^{4} T_{\mathrm{p}}$ in the simulation they deem the most realistic. Neglecting disc dispersal, they estimate growth times of $8000 T_{\mathrm{p}}$. At even this faster rate, our simulations predict that vortex formation would be inhibited. More detailed models of accretion through circumplanetary discs produce similarly low accretion rates, and thus slow growth times. Again, for planet properties akin to Jupiter, studies find $\dot{M}_{\mathrm{p}} \approx 10^{-5} M_{\mathrm{J}} \mathrm{yr}^{-1}$ (e.g. Papaloizou $\&$ Nelson 2005; Ayliffe \& Bate 2009; Rivier et al. 2012). If circumplanetary disc accretion rates were only weakly dependent on planet semimajor axis, this would favour vortex formation at larger disc radii.

Although our calculations above suggest that realistic planet growth times are slow, vortex formation may still be possible with less idealized disc conditions. For example, secondary effects such as the efficient heating of material in the vicinity of an accreting planet (which we neglect in our isothermal discs) have been shown to help vortex formation and survival (Les \& Lin 2015; Lobo Gomes et al. 2015; Owen \& Kollmeier 2016). Even if the inclusion of more rigorous thermodynamics could ameliorate the inhibition of the instability caused by slow planet growth times, vortices induced by slowly-growing giant planets should still be subject to the vortex back-reaction effects that lower their overdensities, widen their azimuthal extents, and shorten their lifetimes as in our study. As discussed in Section 4.2, these characteristics each likely severely damp prospects for observing vortices.

\section{CONCLUSIONS}

In this paper, we explore the impact of a planet's growth time-scale on the properties of vortices induced at the outer gap edge through the RWI. We find that the values of $T_{\text {growth }}$ needed for the formation of long-lived planet-induced vortices are short compared to those expected from core accretion models or mass resupply in a low-viscosity disc. Even with modest values of $T_{\text {growth }} \sim 1000 T_{\mathrm{p}}$, the resulting vortices have lower gas densities, more elongated azimuthal extents, and shorter lifetimes that are less than half their survival times with rapid planet growth. This is in contrast to vortex lifetimes on the order of $10^{3}-10^{4} T_{\mathrm{p}}$ obtained with $T_{\text {growth }} \lesssim 100 T_{\mathrm{p}}$ in previous studies (e.g. Fu et al. 2014a).

With increased planet growth time-scales, we find that the RWI is in fact triggered soon after the planet mass reaches the gap-opening criterion from Crida et al. (2006), which is well below the final planet masses studied here. Although the vortex evolution at the trigger point is not sensitive to $T_{\text {growth }}$, the planet growth time-scale strongly affects the saturated state of the vortex. We attribute the transition to weaker vortex properties to the competition between the time-scale on which the vortex smooths the gap edge as compared to the time-scale on which the growing planet steepens it. We also map out this transition as a function of the dimensionless planet mass growth rate $\dot{q}$, and a dimensionless measure of gapedge steepness $q^{2} \mathrm{Re}$, where $\mathrm{Re}$ is the Reynolds number (Varnière et al. 2004). If the initial vortex forms when the planet is relatively low in mass, the vortex back-reaction on the gap structure is more pronounced, stunting any further growth of the vortex during subsequent planet growth. Conversely, a short $T_{\text {growth }}$ allows the planet to sculpt the gap edge much faster than the vortex can back-react on it.

A unique feature of the vortices induced by slowly-grown planets is their much wider azimuthal extents, which can be twice that of vortices induced by rapidly grown planets. We caution that these elongated vortices (in particular, those induced by lower mass planets) may not survive as long as in our standard resolution simulations, since their lifetimes are halved in our higher resolution simulations. 
If such elongated vortices survive long enough to be observable, we can measure their azimuthal extents from dust observations. Since a planet's growth time-scale preferentially affects the azimuthal extents of the vortex it creates, a measurement of a vortex's azimuthal extent could be used in conjunction with other properties (such as the planet's mass, along with the disc's viscosity and temperature) to provide limits on how quickly the associated planet formed. Other effects that weaken vortices and cause them to be shorter-lived (such as sub-optimal disc temperatures and viscosities, and dust feedback) do not affect their extents nearly as much as the planet's growth rate.

A low-viscosity disc is generally required for planet-induced vortex formation (e.g. de Val-Borro et al. 2007; Fu et al. 2014a). However, the disc viscosity also limits the accretion rate on to the planet, thereby increasing its growth time-scale. In this regime, the corresponding values of $T_{\text {growth }}$ prevent a planet from inducing a long-lived vortex. If the asymmetric discs observed with ALMA are induced by vortices from giant planets, more detailed thermodynamics may need to be incorporated into future simulations to model them properly. Accounting for the disc cooling time-scale or planetary accretion luminosity may lead to stronger vortices even with slow growth rates (Les \& Lin 2015; Lobo Gomes et al. 2015; Owen \& Kollmeier 2016).

Finally, we note that vortices associated with dead zone boundaries (e.g. Regály et al. 2012) are also expected to be elongated. As a result, one would not be able to distinguish the origin of elongated vortices (as induced by a dead zone boundary or a slowly-grown planet) based on azimuthal extent alone.

\section{ACKNOWLEDGEMENTS}

We thank Ruobing Dong, James Owen, Sijme-Jan Paardekooper, Nienke van der Marel, and the anonymous referee for their helpful comments. MH is supported by the NSF Graduate Research Fellowship under Grant No. DGE 1143953. KMK is supported by the National Science Foundation under Grant No. AST-1410174. MKL is supported by the Steward Theory Fellowship. The El Gato supercomputer, which is supported by the National Science Foundation under Grant No. 1228509, was used to run all of the simulations in this study.

\section{REFERENCES}

Andrews S. M., Wilner D. J., Espaillat C., Hughes A. M., Dullemond C. P., McClure M. K., Qi C., Brown J. M., 2011, ApJ, 732, 42

Ayliffe B. A., Bate M. R., 2009, MNRAS, 393, 49

Bae J., Zhu Z., Hartmann L., 2016, ApJ, 819, 134

Barge P., Sommeria J., 1995, A\&A, 295, L1

Birnstiel T., Dullemond C. P., Pinilla P., 2013, A\&A, 550, L8

Casassus S. et al., 2013, Nature, 493, 191

Chiang E. I., Goldreich P., 1997, ApJ, 490, 368

Crida A., Morbidelli A., Masset F., 2006, Icarus, 181, 587

D’Angelo G., Lubow S. H., 2008, ApJ, 685, 560 de Val-Borro M. et al., 2006, MNRAS, 370, 529

de Val-Borro M., Artymowicz P., D’ Angelo G., Peplinski A., 2007, A\&A, 471,1043

Fu W., Li H., Lubow S., Li S., 2014a, ApJ, 788, L41

Fu W., Li H., Lubow S., Li S., Liang E., 2014b, ApJ, 795, L39

Fukagawa M. et al., 2013, PASJ, 65

Joachims T., 1999, in Schlkopf B., Burges C., Smola A., eds, Advances in Kernel Methods - Support Vector Learning, Making Large-scale SVM Learning Practical. MIT Press, Cambridge, MA

Kida S., 1981, J. Phys. Soc. Japan, 50, 3517

Kley W., 1999, MNRAS, 303, 696

Kratter K. M., Lodato G., 2016, ARA\&A, 54, 271

Les R., Lin M.-K., 2015, MNRAS, 450, 1503

Li H., Finn J. M., Lovelace R. V. E., Colgate S. A., 2000, ApJ, 533, 1023

Li H., Colgate S. A., Wendroff B., Liska R., 2001, ApJ, 551, 874

Li H., Li S., Koller J., Wendroff B. B., Liska R., Orban C. M., Liang E. P. T., Lin D. N. C., 2005, ApJ, 624, 1003

Lin M.-K., 2012, MNRAS, 426, 3211

Lin M.-K., Papaloizou J. C. B., 2010, MNRAS, 405, 1473

Lin M.-K., Papaloizou J. C. B., 2011, MNRAS, 415, 1426

Lissauer J. J., Hubickyj O., D’Angelo G., Bodenheimer P., 2009, Icarus, 199,338

Lobo Gomes A., Klahr H., Uribe A. L., Pinilla P., Surville C., 2015, ApJ, 810,94

Lovelace R. V. E., Li H., Colgate S. A., Nelson A. F., 1999, ApJ, 513, 805

Lubow S. H., D’Angelo G., 2006, ApJ, 641, 526

Lyra W., Lin M.-K., 2013, ApJ, 775, 17

Lyra W., Mac Low M.-M., 2012, ApJ, 756, 62

Masset F., 2000, A\&AS, 141, 165

Meheut H., Keppens R., Casse F., Benz W., 2012, A\&A, 542, A9

Meheut H., Lovelace R. V. E., Lai D., 2013, MNRAS, 430, 1988

Miranda R., Li H., Li S., Jin S., 2016, preprint (arXiv:1610.01977)

Muñoz D. J., Kratter K., Springel V., Hernquist L., 2014, MNRAS, 445, 3475

Ono T., Muto T., Takeuchi T., Nomura H., 2016, ApJ, 823, 84

Owen J. E., Kollmeier J. A., 2016, preprint (arXiv:1607.08250)

Papaloizou J. C. B., Nelson R. P., 2005, A\&A, 433, 247

Pérez L. M., Isella A., Carpenter J. M., Chandler C. J., 2014, ApJ, 783, L13

Pollack J. B., Hubickyj O., Bodenheimer P., Lissauer J. J., Podolak M., Greenzweig Y., 1996, Icarus, 124, 62

Regály Z., Juhász A., Sándor Z., Dullemond C. P., 2012, MNRAS, 419, 1701

Rivier G., Crida A., Morbidelli A., Brouet Y., 2012, A\&A, 548, A116

Shakura N. I., Sunyaev R. A., 1973, A\&A, 24, 337

Toomre A., 1964, ApJ, 139, 1217

van der Marel N. et al., 2013, Science, 340, 1199

van der Marel N., van Dishoeck E. F., Bruderer S., Andrews S. M., Pontoppidan K. M., Herczeg G. J., van Kempen T., Miotello A., 2016, A\&A, 585, A58

Varnière P., Quillen A. C., Frank A., 2004, ApJ, 612, 1152

Youdin A. N., Kenyon S. J., 2013, in Oswalt T. D., French L. M., Kalas P., eds, From Disks to Planets. Springer, Dordrecht, p. 1

Zhu Z., 2015, ApJ, 799, 16

This paper has been typeset from a $\mathrm{T}_{\mathrm{E}} \mathrm{X} / \mathrm{L} \mathrm{T} \mathrm{E} \mathrm{X}$ file prepared by the author. 